\section{Public Health Genomics}

Public Health Genomics 2014;17:173-182

DOI: $10.1159 / 000360472$
Received: October 29, 2013

Accepted after revision: February 7, 2014

Published online: June 12, 2014

\title{
Intentions to Donate to a Biobank in a National Sample of African Americans
}

\author{
Jasmine A. McDonald ${ }^{a}$ Susan Vadaparampil ${ }^{b}$ Deborah Bowen ${ }^{c}$ \\ Gayenell Magwood $^{d}$ Jihad S. Obeide, ${ }^{e}$ Melanie Jefferson ${ }^{e, i}$ Richard Drakef,i \\ Mulugeta Gebregziabherg Chanita Hughes Halbert ${ }^{\mathrm{e}}$ i \\ ${ }^{a}$ Department of Epidemiology, Mailman School of Public Health, Columbia University, New York, N.Y., b Moffitt \\ Cancer Center, Tampa, Fla., ' Department of Community Health, Boston University, Boston, Mass., ${ }^{\mathrm{d} C o l l e g e}$ of \\ Nursing, Departments of ${ }^{\mathrm{e}}$ Psychiatry and Behavioral Sciences, ${ }^{f}$ Cell and Molecular Pharmacology and Experimental \\ Therapeutics, and ${ }^{9}$ Public Health Sciences, and hiomedical Informatics Center and 'Hollings Cancer Center, \\ Medical University of South Carolina, Charleston, S.C., USA
}

\section{Key Words}

African Americans · Attitudes · Biobanking

\begin{abstract}
Background/Aims: Despite the investments being made to develop biobanks, African Americans are under-represented in genomic studies. We identified factors having significant independent associations with intentions to donate personal health information and blood and/or tissue samples to a biobank in a national random sample of African Americans $(n=1,033)$. Methods: We conducted a national survey from October 2010 through February 2011. Results: Twenty-three percent of respondents reported that it was not at all likely that they would donate to a biobank, $18 \%$ reported it was a little likely, 36\% reported it was somewhat likely, and 23\% reported it was very likely. Respondents who were likely to donate to a biobank had greater positive expectations about participating in cancer genetics research and reported more participation facilitators relative to barriers. Respondents who were distrustful of researchers had a significantly lower likelihood of being willing to donate to a biobank compared to those who were less distrustful. Conclusions: African Americans have diverse attitudes about participating in ge-
\end{abstract}

netics research, and many are likely to donate to a biobank based on expectations of positive outcomes. It may be important to address attitudes about genetics research as part of recruitment to enhance the quality of informed consent for participation in biobanks among African Americans.

(c) 2014 S. Karger AG, Basel

\section{Introduction}

Biobanks are a critical component of the medical research enterprise and are increasingly being used to support studies that investigate the genetic basis of disease, environmental exposures, and examine the interaction between genetic and environmental factors on chronic health conditions [1-4]. Considerable investments have been made to establish national and local biobanks (e.g. Cancer Human Biobank) [5-7]; financial resources will continue to be invested to support their development and maintenance. Biobanks can be an effective resource for medical research only if research participants donate blood or tissue samples with the associated clinical information and appropriate consenting procedures are in place. Contribution of biospecimens that are collected during routine clin-

\section{KARGER}

E-Mail karger@karger.com

www.karger.com/phg
(C) 2014 S. Karger AG, Basel

$1662-4246 / 14 / 0173-0173 \$ 39.50 / 0$
Chanita Hughes Halbert, $\mathrm{PhD}$

Department of Psychiatry and Behavioral Sciences Medical University of South Carolina, 68 President Street, Suite BE103

Charleston, SC 29425 (USA)

E-Mail hughesha@musc.edu 
ical care for a biobank is considered to be minimally invasive with little risk of physical harm or loss of confidentiality from a regulatory perspective $[8,9]$, especially when identifying information is removed prior to the release of specimens and data to researchers. However, studies have shown that individuals have concerns about the privacy and confidentiality of their health information and potential adverse consequences such as genetic discrimination once their data are included in a biobank [10]. These concerns are more prevalent in some groups; African Americans are more likely than whites to have privacy concerns and are less willing to donate a sample to a biobank as part of ongoing [11] or future research [12, 13].

Racial differences in actual and intended donation to biobanks may be expected given the historical experiences of African Americans in medical research. Recently, Haga [14] found limited racial diversity in the populations included in genome-wide association studies; this will ultimately delay the development of clinical tools that are effective at addressing racial disparities in health outcomes. At the same time, qualitative research has shown that African Americans have some therapeutic misconceptions about providing a biospecimen to a biobank; some believe they would get personal results or clinical care as a result of participating in a biobank [15]. To develop recruitment strategies that are likely to enhance the quality of informed consent for participation in a biobank, it is necessary to identify factors that are likely to be important to participation decisions. But, empirical data on barriers and facilitators to donation intentions in racial minority groups is not available, thereby limiting the development of effective recruitment strategies that enhance the informed consent process, and perpetuating under-representation of certain racial groups in studies that are conducted using biobanks. This report describes the results of a survey conducted in a national, random sample of African American adults $(n=1,033)$ to identify factors significantly associated with intentions to donate personal health information and blood and/or tissue samples to a biobank. Our study was guided by the Theory of Reasoned Action (TRA) and the Theory of Planned Behavior [16], which focus on the relationship between behavioral intentions, attitudes, beliefs, and perceived control. According to the TRA, for instance, behavioral intentions are based in part on an individual's attitudes about that behavior or their beliefs about what positive and negative outcomes will happen as a result of performing the behavior [16]. These theories can be applied to a variety of health behaviors in which individuals are faced with a decision, especially those that depend on beliefs about what will happen as a result of making alternative decisions. In the context of a biobank, individuals have a choice about providing a biospecimen and providing informed consent for this information and clinical health data to be used in research. Our previous research has shown that African Americans would consider a number of ethical, legal and social issues when making a decision to participate in cancer genetics research [17]. We predicted that the likelihood of donating a biospecimen and clinical information to a biobank would be increased with greater expectations about positive outcomes of participating in genetics research and would be reduced with more negative expectations about participating in genetics research and distrust of researchers.

\section{Materials and Methods}

\section{Procedures}

This study was approved by the Institutional Review Boards at the University of Pennsylvania and the Medical University of South Carolina. Study data were collected by a professional survey firm from October 2010 through February 2011 in a random sample of 1,512 African American adults including users of both conventional and cellular phones. Separate lists were used for landlines and cellular phones. To identify households and respondents for the survey, telephone exchanges were randomly selected by a computer from a complete list of more than 69,000 active residential exchanges across the country. The exchanges were chosen to ensure that each region of the country was represented in proportion to its population. Within each exchange, random digits were added to form a complete telephone number, thus permitting access to both listed and unlisted numbers. Numbers for the landline sample were drawn with probabilities in proportion to their share of listed telephone households from active blocks (area code + exchange +2 -digit block number) that contained one or more residential directory listings. The cellular sample was not list assisted, but was drawn through a systematic sampling from dedicated wireless 100 -blocks and shared service 100-blocks with no directory-listed landline numbers. In each household that was reached, one adult was randomly selected to be the respondent for the survey using a standardized procedure. Exchanges with higher concentrations of African Americans were oversampled. To enhance participation in the study, individuals who completed the survey were given a USD 20 incentive. The landline response rate was $31.8 \%$, and the cell phone response rate was $26.8 \%$. Study data were weighted to account for phone service (landline or cellular) and to population targets for education, age and gender, using estimates from the March 2011 Current Population Survey. This analysis includes 1,033 respondents who had complete data on study measures.

\section{Study Measures}

Socioeconomic Factors and Medical History

We obtained socioeconomic characteristics (e.g. age, income, marital status, education level, employment) and health insurance 
status by self-report. We also asked respondents if they had ever been diagnosed with cancer (yes or no) and if they had ever previously participated in a research study (yes or no). Respondents were also asked if they had a family member who had been diagnosed with cancer (yes or no).

\section{Attitudes about Cancer Genetics Research}

We used newly developed instruments to evaluate attitudes about participating in cancer genetics research and reasons for participating in these types of study. The development of these instruments was guided by the TRA, the Theory of Planned Behavior [16], and our formative research which found that African American men and women would consider the potential benefits to themselves, family members and communities when making a decision to participate in cancer genetics research and that concerns about exploitation, distrust, and the investigator's motives would be important to participation decisions [17]. According to the TRA, behavioral intentions are based in part on an individual's attitudes about that behavior, or their beliefs about what positive and negative outcomes will happen as a result of performing that behavior [16]. This model posits that individuals make decisions to engage in behaviors based on their attitudes about the behavior; attitudes are based on one's behavioral beliefs (e.g. if I participate in cancer genetics research, then I will get something beneficial in return) and their evaluation of the outcomes of performing the behavior (e.g. the extent to which individuals view getting something beneficial in return for study participation as being positive or negative) [16]. Based on our qualitative study [17], we developed items that evaluated attitudes about participating in cancer genetics research in terms of expectations about positive and negative outcomes from participating. These items were pilot tested using cognitive interviews with African American men and women through our previous research and had acceptable face validity.

As in other studies that evaluated positive and negative expectations within the context of genetic testing for inherited breast cancer risk $[18,19]$ and limited knowledge about genetic and genomic concepts found among African Americans [20], respondents were read a brief description about the role that genes play in cancer. Participating in cancer genetics research was described as a study that could involve completing a questionnaire about one's medical history, giving a blood, tissue or saliva sample, or receiving education about genetic risk factors. For items that asked about attitudes about participating in cancer genetics research, respondents were asked how much they agreed or disagreed with each statement $(1=$ strongly disagree, $2=$ disagree, $3=$ neutral, $4=$ agree, $5=$ strongly agree) . We summed responses to these items so that higher scores reflected greater expectations about positive outcomes (e.g. the results would be used to help future generations; I would get information about how to detect, prevent, and treat cancer that was just for me) and negative outcomes related to privacy (e.g. my information would not be kept private; the researchers would use the results of the study to make profits), and impact on health care and outcomes (e.g. the results would be used to develop cancer treatment drugs that someone like me could not afford; I would obtain information about my health that I did not want to know). The Cronbach's alpha for these scales were 0.52 for positive expectations, 0.64 for negative expectations about privacy, and 0.55 for negative expectations about the impact on health care and outcomes.
Reasons for Participating in Genetics Research

We also developed items that evaluated important perceptions of behavioral control including the extent to which various aspects of the study (e.g. who was sponsoring the study, logistical aspects of the research and possible outcomes) would make it difficult or easy to participate. These items were also pilot tested using cognitive interviews with African American men and women. We used recommended scoring procedures to characterize this variable [16]. Specifically, it is suggested that control beliefs be measured using a bipolar scale that assesses the likelihood of occurrence. To do this, respondents were asked first how much each issue would affect their decision to participate $(1=$ very likely, $2=$ somewhat likely, 3 = make no difference, 4 = somewhat unlikely, $5=$ very unlikely). Next, responses of 'somewhat or very unlikely' were recoded as -1 , responses of 'make no difference' were recoded as 0 , and responses of 'somewhat or very likely' were recoded as 1 . We then summed the recoded responses to calculate a total score such that a negative score indicated a greater level of barriers that would make study participation more difficult or unlikely. Conversely, a positive score indicated a greater level of facilitators that would make study participation easier or more likely. A score of zero indicated an equal number of barriers and facilitators. Forty-five percent of respondents reported more participation facilitators, $9 \%$ of respondents had an equal number of barriers and facilitators, and $46 \%$ of respondents reported more participation barriers.

\section{Distrust of Researchers}

We adapted an item from our previous research on distrust of health care providers to evaluate distrust of researchers [21]. Respondents were asked how much they can trust researchers to do what is best for participants in their study $(1=$ almost most of the time, 2 = most of the time, $3=$ some of the time, $4=$ almost none of the time). We recoded responses to this item to reflect distrust (some or almost none of the time vs. most or almost most of the time).

Intentions to Donate to a Biobank

We adapted a behavioral intention item from our previous research on informed decision making about genetic testing among African American women to evaluate intentions to donate to a biobank [20]. Specifically, respondents were read the following description: 'Biobanks are now being developed to provide resources to support research on genetic and lifestyle factors involved in diseases. Participating in a biobank would involve giving a blood or saliva sample along with information about your medical history and lifestyle. Your blood or saliva sample would be sent to a lab where a genetic analysis would be completed. Your genetic, medical, and lifestyle information would be stored in a databank. Researchers could apply to use the samples and health information in the databank to study genes, environment, and lifestyle.' This description of a biobank was adapted from a previous study that examined public opinions about privacy in biobank research [13]. After being read this description, respondents were asked how likely they would be to give a blood or saliva sample along with their health information to be included in a biobank $(1=$ not at all likely, 2 = a little likely, 3 = somewhat likely, 4 = very likely). We used a similar type of item to measure intentions to participate in cancer genetics research in a hospital-based sample of African Americans [21]. 
Statistical Analyses

To analyze study data, we first generated descriptive statistics using the weighted data to characterize respondents in terms of socioeconomic factors, health care variables and intentions to donate to a biobank. We then generated a multivariate logistic regression model to identify factors having significant independent associations with intentions to donate biospecimens. As in our previous research $[19,22]$, we recoded intentions to donate to a biobank using 2 strategies to approximate actual donation behaviors. Specifically, the first model compared respondents who reported it was somewhat or very likely that they would donate a biospecimen to a biobank to those who reported not at all or a little likely. The second model compared respondents who reported that it was very likely that they would donate to a biobank to those who reported not at all, a little, or somewhat likely. We included variables that have been associated with intentions to enroll in medical research among African Americans in previous research (e.g. distrust) as well as those that are important to behavioral intentions (e.g. attitudes about participating in cancer genetics research), according to our theoretical model. As described under Procedures, study data were weighted in all analyses to account for phone service and population targets for education, age and gender using estimates from the March 2011 Current Population Survey.

\section{Results}

Table 1 shows the characteristics of our study sample; there was a relatively equal distribution of respondents for most socioeconomic characteristics. For instance, $47 \%$ of respondents were men, $50 \%$ had some college education or were college graduates, and $43 \%$ were employed. However, only $9 \%$ had ever previously participated in a research study and $4 \%$ had a personal history of cancer. The mean (SD) age of respondents was 42.2 (16). Consistent with the geographic distribution of African Americans in the US, most respondents lived in the South (55\%), 18\% lived in the Midwest, $17 \%$ lived in the Northeast, and 10\% lived in the West, using the weighted data.

Table 2 reports descriptive information on items that evaluated attitudes about participating in cancer genetics research and perceptions about participation barriers and facilitators. Items that measured positive expectations about participating in cancer genetics research had a mean endorsement of 4.2-3.4, the mean item endorsement for items that measured negative expectations about privacy ranged from 2.3-2.9, and the mean for items that measured negative expectations about the impact of participating in cancer genetics research ranged from 2.23.4. Table 3 shows responses to reasons for participating in cancer genetics research; the top 3 barriers to participation included: (1) not knowing who would be able to obtain their personal information, (2) difficulty getting to
Table 1. Sample characteristics $(n=1,033)$

n (\%) [weighted data]

$\begin{array}{lc}\text { Gender } & \\ \quad \text { Male } & 478(46)[489.3(47)] \\ \quad \text { Female } & 555(54)[547.7(53)] \\ \text { Marital status } & \\ \quad \text { Married } & 389(38)[409.2(39)] \\ \quad \text { Not married } & 644(62)[627.8(61)] \\ \text { Education level } & \\ \quad \leq \text { High school graduate } & 431(42)[523.4(50)] \\ \quad \text { Some college } & 602(58)[513.5(50)] \\ \text { Employment status } & \\ \quad \text { Employed } & 388(38)[448.2(43)] \\ \quad \text { Not employed } & 645(62)[588.7(57)] \\ \text { Income level } & \\ \quad \text { USD >35,000 } & 489(47)[426.5(41)] \\ \quad \text { USD } \leq 35,000 & 544(53)[610.5(59)] \\ \text { Health insurance } & \\ \quad \text { Yes } & 813(79)[766.9(74)] \\ \quad \text { No } & 220(21)[270.1(26)] \\ \text { Personal history of cancer } & \\ \quad \text { Yes } & 58(6)[42.9(4)] \\ \quad \text { No } & 975(94)[994(96)] \\ \text { Prior study participation } & \\ \quad \text { Yes } & 121(12)[88.8(9)] \\ \quad \text { No } & 912(88)[948.1(91)] \\ \text { Distrust researchers } & \\ \quad \text { Yes } & 543(53)[540.9(52)] \\ \quad \text { No } & 490(47)[496(48)]\end{array}$

where the study was being conducted, and (3) the lack of availability of the findings from the study to the participant. The top 3 facilitators to participating in cancer genetics research or facilitators of participation included if: (1) the study provided free medication or health care, (2) addressed a health condition that was personally relevant, and (3) participation lasted a short period of time. The race of the study investigator, if the study was race specific, and the opinions and advice from family members about participation were the top 3 reasons that had no impact of participation decisions.

Overall, intentions to donate to a biobank were variable; $23 \%$ reported that it was not at all likely that they would donate a blood or saliva sample and provide their health information to a biobank, $18 \%$ reported that it was a little likely, 36\% reported somewhat likely, and $23 \%$ reported very likely. Table 4 shows the results of the multivariate logistic regression model of intentions to donate to a biobank $(\mathrm{F}=3.73, \mathrm{p}=0.0000)$. Of the socioeconomic factors, only age had a significant association with intentions to donate to a biobank. But, several of the atti- 
Table 2. Attitudes about participating in genetics research

Mean \pm SD

Positive

The results would be used to help future generations.

$4.2 \pm 0.78$

I would feel that I contributed to the development of better strategies for detecting, preventing, and treating cancer. $4.0 \pm 0.84$

The results would be used to help people who have a greater chance of developing cancer.

I would get information about how to detect, prevent, and treat cancer that was just for me.

$3.9 \pm 0.93$

$3.4 \pm 1.1$

Summary statistics

$15.5 \pm 2.4$

Negative expectations about privacy

The researchers would use the results of the study to make profits.

$2.9 \pm 1.2$

The researchers would share my personal information with others.

$2.8 \pm 1.2$

My information would not be kept private.

$2.8 \pm 1.2$

Other individuals would be able to access my DNA.

$2.6 \pm 1.2$

My DNA would be tampered with.

$2.3 \pm 1.0$

Summary statistics

$13.4 \pm 3.4$

Negative expectations about impact

The results of the study would be used to develop cancer treatment drugs that someone like me could not afford.

I would obtain information about my health that I did not want to know.

The results of the study would be used to develop ways to detect, prevent, and treat cancer that I would not be able to use.

I would not have any legal rights if something bad happened to me after I enrolled in the study.

The researchers would be dishonest about the purpose of the study.

Summary statistics

$2.2 \pm 0.94$

$16.6 \pm 3.7$

Table 3. Reasons for participating in cancer genetics research: facilitators and barriers

Unlikely, \% Neutral, \% Likely, \%

If I did not know who would be able to obtain my personal information

(e.g. blood or saliva sample, medical history).

If it were hard for me to get to where the study was being conducted.

If findings from the study would not be made available to me.

If I would get free medication or health care.

If the study addressed a health condition I was worried about.

If I had to participate in the study for a short period of time.

If someone from my racial group was conducting the study.

If the study only included African Americans.

If my family members or friends did not think I should participate in the study.

If my family members or friends told me not to participate in the study.

If I had to participate in the study for a long period of time.

If the results of the study would be used to develop drugs I might not be able to afford.

If I did not receive any financial compensation for my time.

If the study were sponsored by a pharmaceutical company.

If I were afraid of getting information about my health that I did not want to know. $\quad 34$

If my information (e.g. medical history, blood or saliva sample) would

be used in other studies.

$\begin{array}{lll}\mathbf{6 4} & 18 & 18 \\ \mathbf{6 3} & 14 & 23 \\ \mathbf{6 0} & 21 & 19 \\ 10 & 21 & \mathbf{6 9} \\ 15 & 19 & \mathbf{6 6} \\ 15 & 25 & \mathbf{6 0} \\ 12 & \mathbf{5 6} & 32 \\ 26 & \mathbf{4 4} & 30 \\ 36 & \mathbf{4 0} & 23 \\ 38 & \mathbf{4 0} & 22 \\ 48 & 20 & 31 \\ 42 & 27 & 31 \\ 36 & 38 & 26 \\ 28 & 36 & 36 \\ 34 & 32 & 35 \\ 33 & & 38\end{array}$

Numbers in bold type show items with the greatest endorsement. 
Table 4. Multivariate logistic regression model of intentions to donate to a biobank

\begin{tabular}{|c|c|c|}
\hline & $\begin{array}{l}\text { Somewhat/very likely vs. else, } \\
\text { OR (95\% CI) }\end{array}$ & $\begin{array}{l}\text { Very likely vs. else, } \\
\text { OR }(95 \% \mathrm{CI})\end{array}$ \\
\hline \multicolumn{3}{|l|}{ Gender } \\
\hline Male/female & $0.78(0.55-1.11)$ & $0.78(0.53-1.15)$ \\
\hline \multicolumn{3}{|l|}{ Marital status } \\
\hline Married/not married & $1.18(0.81-1.72)$ & $1.38(0.89-2.12)$ \\
\hline \multicolumn{3}{|l|}{ Education level } \\
\hline$\geq$ Some college/ $\leq$ high school graduate & $1.17(0.80-1.72)$ & $0.78(0.52-1.15)$ \\
\hline \multicolumn{3}{|l|}{ Employment status } \\
\hline Employed/not employed & $1.30(0.88-1.93)$ & $0.98(0.63-1.52)$ \\
\hline \multicolumn{3}{|l|}{ Income level } \\
\hline USD $>35,000 / \leq 35,000$ & $0.87(0.57-1.34)$ & $0.99(0.63-1.58)$ \\
\hline \multicolumn{3}{|l|}{ Age } \\
\hline Continuous $^{\mathrm{a}}$ & $1.20(1.00-1.45)^{*}$ & $1.42(1.18-1.71)^{* * *}$ \\
\hline \multicolumn{3}{|l|}{ Health insurance } \\
\hline Yes/no & $0.74(0.47-1.17)$ & $0.72(0.44-1.18)$ \\
\hline \multicolumn{3}{|l|}{ Personal history of cancer } \\
\hline Yes/no & $0.70(0.37-1.34)$ & $0.96(0.45-2.07)$ \\
\hline \multicolumn{3}{|l|}{ Prior study participation } \\
\hline Yes/no & $1.14(0.66-1.95)$ & $0.87(0.51-1.49)$ \\
\hline \multicolumn{3}{|l|}{ Distrust } \\
\hline Yes/no & $0.62(0.44-0.87)^{* *}$ & $0.41(0.28-0.60)^{* * *}$ \\
\hline \multicolumn{3}{|l|}{ Positive expectations } \\
\hline Continuous $^{\mathrm{a}}$ & $1.33(1.12-1.57)^{* * *}$ & $1.27(1.02-1.58)^{*}$ \\
\hline \multicolumn{3}{|l|}{ Negative expectations about privacy } \\
\hline Continuous $^{\mathrm{a}}$ & $0.92(0.75-1.12)$ & $0.76(0.59-0.98)^{*}$ \\
\hline \multicolumn{3}{|l|}{ Negative expectations about impact } \\
\hline Continuous ${ }^{\mathrm{a}}$ & $1.00(0.81-1.23)$ & $1.15(0.92-1.44)$ \\
\hline \multicolumn{3}{|l|}{ Participation barriers and facilitators } \\
\hline Facilitators vs. barriers & $2.17(1.51-3.10)^{* * *}$ & $1.69(1.15-2.48)^{* *}$ \\
\hline Equal facilitators/barriers vs. barriers & $1.76(0.92-3.36) \ddagger$ & $1.80(0.80-4.05)$ \\
\hline
\end{tabular}

tudinal and behavioral control variables had significant associations with intentions. Respondents with more positive expectations about the outcomes of participating in genetics research had a significantly greater odds of being willing to donate a blood or tissue sample and their health information to a biobank. In addition, respondents who were distrustful of researchers had a significantly lower odds of being willing to donate a sample or health information to a biobank compared to those who were less distrustful. Reasons for participating in cancer genetics research were also associated with intentions to donate to a biobank, but only the comparison for facilitators versus barriers was significant. Respondents who had more participation facilitators compared to barriers were about twice as likely to be willing to donate a sample or health information to a biobank. Negative expectations about privacy and the impact of cancer genetics research did not have significant associations with intentions to donate. The results were unchanged in the model $(\mathrm{F}=4.91, \mathrm{p}=$ 0.0000 ) with intentions coded as very likely versus else, with the exception that the association between negative expectations about privacy and intentions to donate was significant (table 4). The odds of being very likely to donate a biospecimen or health information decreased as these negative expectations increased.

\section{Discussion}

The purpose of this study was to characterize intentions to donate biospecimens to a biobank in a national sample of African Americans and to identify variables 
significantly associated with this behavior. Developing a better understanding of intentions to donate to a biobank based on attitudes and barriers and facilitators in this population is important to identify factors that are likely to promote or hinder participation so that recruitment strategies that enhance the provision of informed consent can be developed. We found that the likelihood of donating a biospecimen and clinical health information to a biobank was increased with greater age, more positive expectations about participating in genetics research, and more participation facilitators relative to barriers.

Our study provides novel empirical data on the likelihood of donating a biospecimen and clinical health information in this population. Therefore, it extends results of qualitative studies that examined beliefs and attitudes about genomic research and biobanks in geographically homogenous samples and those from single academic institutions $[10,17,23]$. An additional strength of our study is that we used an established theoretical model as the framework for understanding behavioral intentions and developing study measures [16]. These innovative features of our study should be considered within the context of a limited response rate, despite sampling from both cellular and landlines. Diminishing response rates is an issue across all types of survey research; the Pew Research Center has shown that their response rate for telephone surveys has declined from 36\% in 1997 to $9 \%$ in 2012 [24]. Despite declining response rates, the samples enrolled in telephone surveys are likely to be similar to the US population in terms of demographics and other variables [24]. This increases our confidence that the estimates we obtained are not likely to be biased because of nonresponse. Additional limitations may be that we measured behavioral intentions and distrust using a single item, subjective norms were not measured as a separate scale, and the items that measured attitudes about participating in cancer genetics research had modest reliability estimates. Behavioral intentions are conceptualized as the best predictor of actual behavior; the similarities we observed between genetic testing intentions [19] and actual uptake rates of $B R C A 1$ and $B R C A 2(B R C A 1 / 2)$ mutation testing among African American women [25] suggests that intentions are a reasonable proxy for behavior in this population. Also, the influence of others, which is conceptually similar to subjective norms, was captured in the items we used to measure participation facilitators and barriers. Nonetheless, additional research is needed to evaluate the relationship between intentions to donate to a biobank and actual donation behaviors. It may also be important to measure behavioral intentions and dis- trust using multiple questions rather than a single item. Future studies are also needed to address the reliability of the items we developed to measure attitudes about participating in cancer genetics research. Despite having acceptable face validity, these items had modest internal consistency in the present sample even though they were developed through focus groups with African Americans and pilot tested using cognitive interviews. Lastly, the focus of this study was on African Americans, since previous research has shown that they are less likely to provide consent for biospecimens to be used in future research $[11,26]$ and are under-represented in genome-wide association studies that often rely on biobanks to provide samples and associated clinical data [14].

We found that the pattern of responses for donation intentions mirror actual participation rates in some cases, but are lower than the donation rates observed in other settings. For instance, only 15\% of African American women enrolled in a cancer genetics registry [27], and we found that $23 \%$ of respondents would definitely donate a sample. We combined responses of somewhat and definitely likely in our regression model; this corresponds to a $59 \%$ likely donation rate, which is closer to, but lower than actual consent rates of $73.2 \%$ among African Americans to have biologic samples and DNA stored in the NHANES national repository for genetic research [11, 26]. Regardless of how we categorized intentions to donate to a biobank, the same variables had significant independent associations with donation intentions. We found that respondents who were older in age, were not distrustful of researchers, and had more positive expectations about participating in cancer genetics research were most likely to be willing to donate a biospecimen and health information to a biobank. In addition, respondents who had more participation facilitators relative to barriers were significantly more likely to be willing to donate to a biobank. Negative expectations about the impact of participating in cancer genetics research were not associated with intentions to donate to a biobank in either model, and concerns about privacy were only associated significantly in the model that categorized donation intentions as very likely versus other responses. These findings suggest that despite African Americans' concerns about privacy and confidentiality of their health information and the possibility of adverse consequences of donating their health information to a biobank [10-13], these concerns may not influence the decision to participate in this type of research. In light of these findings, it is curious that negative expectations about participating in cancer genetics research were not associated with a reduced odds 
of being willing to participate in a biobank, except for in the model in which donation intentions were categorized as very likely versus else. It may be that concerns about biobanking are reflected in more general beliefs about the trustworthiness of the researchers conducting the study. Respondents who were distrustful of researchers had a significantly lower odds of being willing to donate to a biobank compared to those who trusted investigators. Data from qualitative studies with patients have demonstrated that preferences for using a broad informed consent process in biobank research, or obtaining general consent to participate in biobanks without obtaining subsequent consent to use specimens in future research, are based on an assumption that institutions and researchers will use the samples ethically and that the biospecimens that are donated will be used to advance scientific discovery and medical care [23]. About half of the respondents in this study did not trust researchers; it may be important to understand how attitudes, participation barriers and facilitators, and informed consent preferences for biobanks differ based on levels of distrust in researchers and health care systems.

We also examined attitudes and reasons for participating in cancer genetics research. While logistical factors, privacy concerns and access to study findings were the top 3 barriers to participation, respondents who had more participation facilitators were more likely than those with barriers to be willing to donate a biospecimen. The most important reasons for participating in a cancer genetics study were: if free medication or health care would be provided, if study participation lasted a short period of time, and if the study addressed a health issue or condition that was important to them. Recent qualitative research has shown that African Americans have some therapeutic misconceptions about providing a biospecimen to a biobank; some participants in a deliberative engagement study believed that they would get personal results or clinical care as a result of participating in a biobank [15]. Researchers may obtain informed consent for biobank specimens from patients receiving clinical care and individuals participating in research studies; our findings suggest that individuals with positive expectations about participating in genetics research may be more likely to participate in a biobank. To the extent that individuals conflate their clinical care and/or research benefits with participation in a biobank may indicate a need to draw a greater distinction between clinical care and research and biobank studies during the informed consent process in order to avoid therapeutic misconception. Additional research is needed to evaluate the quality of informed consent for biobank participation and identify factors associated with poor quality decisions about providing a biospecimen and clinical health data so that strategies can be developed to address these deficits. However, our results show that the positive expectations about participating in cancer genetics that were altruistic in nature, such as benefits to future generations, were associated with donation intentions, whereas negative expectations about privacy were not related to intentions. This suggests participants may still be willing to participate in biobanks even if it is made clear during consent that there is no direct benefit to them individually.

Our findings have important implications for African American participation in biobanks and the factors that these individuals are likely to consider as part of making a decision to donate biospecimens and their clinical health information. First, the results show that many African Americans are likely to consider donating a biospecimen and their clinical health information to a biobank, and a sizeable minority is definitely likely to participate. Previously, we found that hospital-based resources were most likely to be used to recruit participants into research on BRCA1 and BRCA2 mutations [28]; it could be that the limited representation of African Americans in genomic studies is due to lack of racial diversity in the clinical settings in which biobanks are being established. This suggests a possible need for efforts to expand recruitment efforts to clinical settings that serve a more heterogeneous population in order to ascertain biospecimens and clinical health data from under-represented groups. To our knowledge, community-based approaches have not been used to increase the diversity of biobanks. This could be an important strategy but should be developed and implemented collaboratively with community stakeholders. Another possibility is that African American patients are not invited to participate in a biobank at the same rate as other patients, or the process for obtaining informed consent is not effective for these patients. Empirical data are needed to understand how system- and patient-level factors influence decisions to participate in a biobank so that these issues can be addressed in ways that are consistent with the fundamental principles of informed consent for genomic research.

African Americans bear a disproportionate burden of morbidity and mortality associated with many chronic conditions that are currently the focus of genomic study (e.g. cardiovascular disease, cancer). The future of genomic research to address racial disparities is highly dependent on obtaining biospecimens from a diverse group of patients and healthy individuals. Previous reports have 
described different ways in which adequate representation of racial minorities can be achieved [29, 30]; this is an important issue in genomic research because in most cases, genetic variants associated with chronic diseases are first identified and validated in samples from white adults, and then replication studies are conducted in other populations. This approach is used to control for complex issues such as population stratification, but is also necessary because racial minorities are not likely to be represented in existing datasets and cohorts at the same rates as whites $[14,27]$. Increasing the representation of racial minorities in biobanks would ensure that a sufficient number of biospecimens are available to conduct subgroup analyses; this would enhance the discovery of genomic risk factors and the translation of these findings into clinical care to reduce racial disparities in health outcomes. This study identifies several factors that can inform future recruitment efforts to increase African American participation in biobanks. Public health practitioners can contribute to this process by working as part of teams to develop strategies that help to ensure the quality of informed consent, facilitate understanding about how the information will be used, provide feedback about the results of the research from the biobanks, and offer participation in the biobanks along with a tangible service that is valued and addresses a health need or concern. These efforts may be effective at increasing the number of African Americans who express intention and ultimately donate to biobanks.

\section{Acknowledgements}

This research was supported by the National Human Genome Research Institute grant R01HG004346. We would like to acknowledge ABT/SBRI for conducting telephone interviews, and Benita Weathers, MPH, and Aliya Collier, BA, for assistance with project and data management. We are very grateful to the men and women who participated in this research.

\section{References}

1 Anton-Culver H, Ziogas A, Bowen D, Finkelstein D, Griffin C, Hanson J, Isaacs C, KastenSportes C, Mineau G, Nadkarni P, Rimer B, Schildkraut J, Strong L, Weber B, Winn D, Hiatt R, Nayfield S: The Cancer Genetics Network: recruitment results and pilot studies. Community Genet 2003;6:171-177.

2 Austin MA, Harding S, McElroy C: Genebanks: a comparison of eight proposed international genetic databases. Community $\mathrm{Ge}-$ net 2003;6:37-45.

3 Kaiser J: Genomic medicine. African-American population biobank proposed. Science 2003;300:1485

-4 Mailman MD, Feolo M, Jin Y, Kimura M, Tryka K, Bagoutdinov R, Hao L, Kiang A, Paschall J, Phan L, Popova N, Pretel S, Ziyabari L, Lee M, Shao Y, Wang ZY, Sirotkin K, Ward M, Kholodov M, Zbicz K, Beck J, Kimelman M, Shevelev S, Preuss D, Yaschenko E, Graeff A, Ostell J, Sherry ST: The NCBI $\mathrm{dbGaP}$ database of genotypes and phenotypes. Nat Genet 2007;39:1181-1186.

5 Massett HA, Atkinson NL, Weber D, Myles R, Ryan C, Grady M, Compton C: Assessing the need for a standardized cancer HUman Biobank (caHUB): findings from a national survey with cancer researchers. J Natl Cancer Inst Monogr 2011;8-15.

-6 Shaw PM, Patterson SD: The value of banked samples for oncology drug discovery and development. J Natl Cancer Inst Monogr 2011; 46-49.
7 Vaught J, Rogers J, Myers K, Lim MD, Lockhart N, Moore H, Sawyer S, Furman JL, Compton C: An NCI perspective on creating sustainable biospecimen resources. J Natl Cancer Inst Monogr 2011;1-7.

8 National Bioethics Advisory Commission: Research Involving Human Biological Materials: Ethical Issues and Policy Guidance, vol 1. Rockville, National Bioethics Advisory Commission, 1999.

9 U.S. Department of Health and Human Services: Summary of the HIPAA Privacy Rule. 2003. www.hhs.gov/ocr/privacy/hipaa/understanding/summary/index.html.

10 Lemke AA, Wolf WA, Hebert-Beirne J, Smith ME: Public and biobank participant attitudes toward genetic research participation and data sharing. Public Health Genomics 2010; 13:368-377.

11 McQuillan GM, Pan Q, Porter KS: Consent for genetic research in a general population: an update on the National Health and Nutrition Examination Survey experience. Genet Med 2006;8:354-360.

12 Bussey-Jones J, Garrett J, Henderson G, Moloney M, Blumenthal C, Corbie-Smith G: The role of race and trust in tissue/blood donation for genetic research. Genet Med 2010;12:116121.

13 Kaufman DJ, Murphy-Bollinger J, Scott J, Hudson KL: Public opinion about the importance of privacy in biobank research. Am J Hum Genet 2009;85:643-654.

14 Haga SB: Impact of limited population diversity of genome-wide association studies. Genet Med 2010;12:81-84.
15 Halverson CM, Ross LF: Incidental findings of therapeutic misconception in biobankbased research. Genet Med 2012;14:611-615.

16 Montano DE, Kasprzyk D: Theory of reasonsed action, theory of planned behavior, and the integrated behavioral model; in Glanz K, Rimer B, Viswanath K (eds): Health Behavior and Health Education. Theory, Research, and Practice. San Francisco, John Wiley \& Sons, 2008, pp 67-96.

17 McDonald JA, Barg FK, Weathers B, Guerra CE, Troxel AB, Domchek S, Bowen D, Shea JA, Halbert CH: Understanding participation by African Americans in cancer genetics research. J Natl Med Assoc 2012;104:324-330.

18 Hughes C, Gomez-Caminero A, Benkendorf J, Kerner J, Isaacs C, Barter J, Lerman C: Ethnic differences in knowledge and attitudes about $B R C A 1$ testing in women at increased risk. Patient Educ Couns 1997;32:51-62.

19 Kessler L, Collier A, Brewster K, Smith C, Weathers B, Wileyto EP, Halbert CH: Attitudes about genetic testing and genetic testing intentions in African American women at increased risk for hereditary breast cancer. Genet Med 2005;7:230-238.

20 Kessler L, Collier A, Halbert CH: Knowledge about genetics among African Americans. J Genet Couns 2007;16:191-200.

21 Halbert CH, Armstrong K, Gandy OH Jr, Shaker L: Racial differences in trust in health care providers. Arch Intern Med 2006; 166: 896-901 
22 McDonald JA, Weathers B, Barg FK, Troxel $A B$, Shea JA, Bowen D, Guerra CE, Halbert $\mathrm{CH}$ : Donation intentions for cancer genetics research among African Americans. Genet Test Mole Biomarkers 2012;16:252-258.

23 Lemke AA, Halverson C, Ross LF: Biobank participation and returning research results: perspectives from a deliberative engagement in South Side Chicago. Am J Med Genet 2012; 158A:1029-1037.

24 Pew Research Institute: Assessing the representativeness of public opinion surveys. 2012. http://www.people-press.org/2012/05/15/assessing-the-representativeness-of-publicopinion-surveys/.
25 Halbert CH, Kessler L, Stopfer JE, Domchek $S$, Wileyto EP: Low rates of acceptance of $B R C A 1$ and BRCA2 test results among African American women at increased risk for hereditary breast-ovarian cancer. Genet Med 2006;8:576-582.

26 McQuillan GM, Porter KS, Agelli M, Kington $\mathrm{R}$ : Consent for genetic research in a general population: the NHANES experience. Genet Med 2003;5:35-42.

27 Moorman PG, Skinner CS, Evans JP, Newman B, Sorenson JR, Calingaert B, Susswein L, Crankshaw TS, Hoyo C, Schildkraut JM: Racial differences in enrolment in a cancer genetics registry. Cancer Epidemiol Biomarker Prev 2004;13:1349-1354.
28 Hughes C, Peterson SK, Ramirez A, Gallion KJ, McDonald PG, Skinner CS, Bowen D: Minority recruitment in hereditary breast cancer research. Cancer Epidemiol Biomarker Prev 2004;13:1146-1155.

29 National Institutes of Health: NIH Guideline on the Inclusion of Women and Minorities as Subjects in Clinical Research. http:/grants. nih.gov/grants/funding/women_min/guidelines_update.htm.

30 National Heart, Lung, and Blood Institute: Questions and Answers on Inclusion of Minorities and Women in Study Populations. http://www.nhlbi.nih.gov/funding/policies/ nhlbigui.htm. 